Increase of the heat at this stage is not very common with young infants; it persists about the same degree, or even decreases.

Whenever continued fever takes this course, the danger is great, and if again, in the space of twentyfour hours, these symptoms do not change for the better, you may in most cases expect a fatal end, under a more or less rapid exhaustion of vital action, occasionally in a fit of eclampsy. In other cases the asthenic stage takes a slower and longer course, sometimes passing into a chronic and weary remittent form, ending in gradual recovery or death.

In bodies of young children, dead within that stage and time, we are far more frequently than in adults at a loss to find remarkable anatomical changes, unless you would qualify as such the variable congestions, of an active or passive character, in one part or other, or partia! injections of the mucous membrane, or effosions in one or the other of the cavities, or many kinds of complicated affections. An this I saw, but variable and inessential. The blood seems not to reach in children, in acute fevers, to that degree of chemical alteration, that it does in adults, and the whole course of the fever is too short to effect such marked anatomical changes. The mentioned slower asthenic forms affect more considerably the tissues; but this is not to our present purpose. Next I will speak of the treatment.

\section{A FATAL CASE OF HYDROPHOBIA} TREATED WITH CHLOROFORM.

\section{By THOMAS SANDWITH, M.D., Beverley.}

Whether chloroform will prove to be an antidote for hydrophobia remains to be seen, and is an event rather to be desired than expected; but that it will procure an enthanasia is certain. In order to prevent speedy death from the exhausting effects of the spasms of the throat, and allow time for the elimination of the morbid poison in hydrophobia, it has been proposed to open the traches. It would, however, appear from the following case that all the advantages that could be derived from tracheotomy may be obtained from chloroform, and these in a manner far more agreeable to the patient, as well as the medical attendant.

William Warden, aged 42 years, was attacked by a strange dog, and one of the fingers of the left hand was wounded, about six months prior to the outbreak of the symptoms of hydrophobia. The dog was immediately destroyed, and the wound healed so quickly that in the course of two or three days he was able to resume his occupation as a labourer in a tan-yard. On the 4th of October he applied to one of the medical officers of the dispensary, on account of a pain in the left side, which was supposed to be rheumatic, and was treated accordingly. In the evening of that day symptoms of hydrophobia made their appearance, and the following morning he was visited by another medical man, who bled him from the arm to a moderate extent. The paroxysms increasing in frequency and violence during the day, in his struggles the bandage became loose, and he lost a very large quantity of blood. $\mathbf{B y}$ this untoward event, and the exhaustion produced by his struggles to recover his breath, when nearly strangled by the spasmodic contractions of the muscles of the larynx, his strength was greatly reduced. His struggles during a paroxysm were said to be terrific, and it required the strength of four men to keep him down in bed.

Through the kindness of Mr. Brandon, jun., I saw this patient at eight o'clock, P.M., and remained at his bedside some hours. He was raving like a maniac, and was in a state of extreme debility. The face was pale, and the skin covered with a cold clammy perspiration; the pupils of the eyes completely dilated; the corners of the mouth retracted; the breathing hurried; the pulse 120 in a minute, thready and vermicular; and the paroxysms produced by the spasms of the throat terrible to behold. The head was on these occasions drawn backward, and he made the most vehement efforts to get out of bed, foam issuing from his mouth profusely.

Some chloroform had been administered during the afternoon. I recommended it to be repeated, and had the satisfaction of witnessing its effects, which were almost magical. He was no sooner under the influence of this potent spell than his respiration became perfectly tranquil ; some colour returned to his cheeks; the pupils cuntracted; the pulse became calm, and, considering his weak state, well developed, the number of pulsations in a minute not exceeding 84 ; and he lay in a state of happy delirium. A more pleasing transition from extreme agony to tranquil ease I never witnessed before. The effect of the remedy was maintained during the night by the occasional use of small doses; but he died the following morning, without pain or agony.

It must be admitted that this was not a favourable case to test the power of chloroform as an antidote. The unhappy patient had lost so much blood as almost to induce me to believe that his death must be set to the account of phlebotomy, rather than to hydrophobia. But on the supposition that we are not yet in possession of a specific for this frightful malady, which is too probable, it must be a gratification to every humane mind to know that it is in our power to alleviate the dreadful sufferings peculiar to hydrophobia, and render the last hours of an unhappy patient tranquil, and free from pain.

In the case of hydrophobia related by Mr. Hunter, he says:- "The pulse in the beginning was not quick, nor was the skin hot; and there was none of the muscular debility so remarkable in fever;"* and Dr. Catrie, of Liverpool, who saw five cases, says :- "In none was there any sense of animal heat." $\dagger$ It is clear,

- Transactions of the society for Promoting Medical Knowledge. Vol. I., p. 308

† Medical Reports. Vol. I., p. 178. 
therefore, that the disease is not inflammatory, and blood-letting is contraindicated. There appears, indeed, to be an affinity between this disease and its congeners,-hysteria, mania, and tetanus, in none of which th the:e an increase of animal heat. Galen calls mania the "delirium sine febre;" and according to Dr. Beddoes " it is certain that tetanus exists without increased heat;"* which conclusion is supported by the experiments of Dr. Currie, whose remarks on the impropriety of blood-letting in tetanus are equally applicable in hydrophobia.

"It is deeply to be lamented," says this able pathologist, "that this disease should ever have been considered as of an inflammatory nature, and that there are even now physicians who treat it by venesection. It is in my mind decisive against this supposition that though the general system is so powerfully affected, the animal heat is not increased, which it uniformly is in all cases where there is an inflammatory affection of the system, whether originating or terminating in local phlegmonic inflammation." +

Opium has always failed to give relief in hydrophobia ; and I cannot think favourably of large doses of arsenic, as recommended by Dr. Billing. We may, however, hope that as we have obtained a remedy for the painful symptoms of this distressing malady, an antidote will yet be discovered. An eternal debt of gratitude is due to Dr. Simpson for his discovery of the anæsthetic properties of chloroform, which, if administered, with the cautions and restrictions laid down by Mr. Nunneley, of Leeds, $\ddagger$ can seldom be dangerous.

Beverley, June 29, 1852.

\section{REMARK8}

ON THE EFFECTS OF IODINE ON"THE GLANDULAR SYSTEM, AND ON THE PBUPERTIES OF KOUSSO.

B. ThOMas H. Silvester, M.D., Claphay.

\author{
Fead al the Anniverwary Meeting of the Bouth-Bastern Branch.
}

Or the Eptects of Ioding on the Glandular

Is our journal the question has been asked,-Whether atrophy or absorption ever takes place in the glandular system from the use of iodine? In answer to this question, 1 would beg the favour of the present members of the Society to allow me to make a few remarks, the result of many years' attention to this point. From 1834 to 1844 a great many patients, suffering under secondary or tertiary syphilis, were admitted into St. Thomas's Hospital, more especially under the care of the late Dr. Williams, who had gained a high reptita-

\footnotetext{
- Daddoes on Fever, p. 30.

+ Medieal Reports. Vol. II., p. 173.

Tol XVI. P. 377.
}

tion in the treatment of these morbid symptoms. Most of these patients came under my notice and particular: observation, and many of the remarkable cases were entered in my note-book, but not one instance of atrophy or absorption of the large glands, occurred in our experience. It was thought advisable, on the recommendation of Lugol, to test the efficacy of the iodide of potassium in scrofulous enlargement of the glands, and in order to give M. Lugol's method of treatment fair play, a most characteristic specimen of these affections was selected. A young woman, fat, florid, and fair, aged 18, was admitted with suppurating glands at the angle of the jaw, and others approaching suppuration or hard and inflamed, extending to the chin, were conspicuously prominent. Eight grains of the iodide of potassium, in camphor mixture, were prescribed, and steadily administered, for nearly six months, without the slightest perceptible effect upon the scrofulous mass of glands, and she was presented in much the same state as at her admission. Now, it happened that in this girl the breasts were largely developed, but no. change was produced in their size by the treatment adopted for the scrofulous ailment, notwithstanding the full dose, and prolonged administration of the iodide.

There were at this period, before the treatment had become generally known, innumerable cases of syphilitic periostitis in which the iodide of potassium was very successful, and yet we never witnessed atrophy or absorption of either the breast or testicle during the use of this remedy. A case of simple hypertrophy of the breasts was then made the subject of experiment; eight grains of the drug were taken, steadily and continuously, for three months, but no diminution of the mammæ took place.

A boy, aged 12, presented limself with immensely enlarged tonsils, and took the iodide nearly six months, without any impression having been made upon theseorgans. It would weary you to bring forward further illustration on this subject, and this negative kind of argument is, I am aware, not perfectly satisfactory, and may be destroyed by a single example of the positive power of the remedy in causing absorption of either the breast or testicle; but ten' years' observation in a large hospital failed to furnish me with a single proofin favour of the opinion, that atrophy or absorption of the glandular system, in its normal condition, arises from the use of iodine in any form. Experience as to the topical application of this powerful agent, involves an inquiry into the effects of friction, etimulation, protection, and warmth, and excludes oll inference as to its: specific property. It must be confessed, that enlarged testicles not unfrequently yield to its influence, but ib will be found on inquiry that in these cases the system. has been contaminated by the syphilitic poison. The same remark is applicable to chronic induration of the inguinal glands. It is a very remarkable fact, that the swelling of the thyroid body, in common bronchocele, vanishes under the internal use of iodine, especially the iodide of potassium. The rapidity and certainty of its. removal are equalled only by that of the venereal node; and I have sometimes thought that there may be a vital 